\title{
Interstitial brachytherapy technique for chest wall refractory recurrence of breast cancer
}

\author{
Ning Wu, MD*, Qianqian Chen, MM*, Zhipeng Zhao, MS, Hongfu Zhao, MS, Guanghui Cheng, MD, PhD \\ *These two authors contributed equally to this work. \\ Department of Radiation Oncology, China-Japan Union Hospital of Jilin University, Changchun, China
}

\begin{abstract}
Purpose: To report the treatment effect of interstitial brachytherapy for chest wall locoregional recurrence of breast cancer.

Material and methods: This 44-year-old female presented with chest wall recurrence seven years after modified radical mastectomy for stage II breast cancer. Despite external beam radiation and chemotherapy, the lesion expanded as $5.3 \times 5.1 \times 3.0 \mathrm{~cm}^{3}$, and $8.0 \times 5.1 \times 4.0 \mathrm{~cm}^{3}$. The locoregional recurrent tumor was treated with interstitial brachytherapy under ultrasound guidance. The brachytherapy dose was $30 \mathrm{~Gy}$ in 6 fractions of $5 \mathrm{~Gy}$ each.

Results: Removal of the recurrent tumor was securely achieved by interstitial brachytherapy guided with ultrasound scanning. The refractory tumor in patient healed uneventfully after interstitial brachytherapy without recurrence during the 7 months of follow-up.

Conclusions: The ultrasound-guided interstitial brachytherapy may be effective for refractory recurrence of breast cancer.

J Contemp Brachytherapy 2015; 7, 4: 290-294 DOI: $10.5114 /$ jcb.2015.53868
\end{abstract}

Key words: breast cancer, interstitial brachytherapy, refractory recurrence.

\section{Purpose}

Breast cancer is one of the most common malignant tumor in female carcinomas. Breast cancer (I, IIA, IIB, and IIIA) is usually treated with surgery, which may be followed by chemotherapy or radiation therapy, or both $[1,2]$. Radiation therapy could be delivered as external beam radiotherapy or as brachytherapy. Brachytherapy to treat breast cancer is usually performed with high-doserate (HDR) temporary brachytherapy. Post surgery, breast brachytherapy could be used as a "boost" following irradiation of the whole breast using external beam radiotherapy (EBRT) [3-5].

Locoregional recurrence in women with breast cancer should undergo surgical resection of the local recurrence, involved-field radiotherapy if it may be safely administered and limited duration systemic chemotherapy or hormonal therapy. The therapeutic researches emphasized the importance of individualizing treatment strategies in patient with a recurrence of disease limited to a local site. In this context, we propose a technique using HDR interstitial brachytherapy to treat refractory recurrence of breast cancer after conventional radiochemotherapy.

\section{Case report}

The case of a 44-year-old female with chest wall recurrence of breast cancer after initial surgery and radiochemo- therapy is presented. Seven years before admitting to our clinic, she visited a vicinity clinic with a lump $(2.4 \times 2.2 \times$ $3.0 \mathrm{~cm}^{3}$ ) located in the upper-outer quadrant of her right breast. She was diagnosed with breast cancer $\left(\mathrm{T}_{2} \mathrm{~N}_{1} \mathrm{M}_{0}\right.$; IIB stage) and received modified radical mastectomy; pathological result revealed medullary carcinoma and positive for ER, PR, and CerbB-2 expressions. The Ki-67 index was $30 \%$ based on the proportion of positively-stained tumor cells. Adjuvant chemotherapy with AC (adriamycin + cyclophosphamide) $\times 4$ followed by T (paclitaxel) $\times 3$, and hormonal therapy with tamoxifen were immediately started. The patient underwent initial radiotherapy by external beam: a total of 50 Gy in 2 Gy fractions for the chest wall and supraclavicular field. She had an abnormal CT scan revealing an area of distortion of approximately $3.0 \times 3.0 \times 2.3 \mathrm{~cm}^{3}$ in size on the skin surface of right chest wall, which was biopsied, and fine-needle aspiration was positive for malignant tumor (originate from breast) dating back to 18 months ago. The proposal of surgery was rejected by patient. She received 3 cycles of chemotherapy with GP (gemcitabine + cisplatin) and chose to undergo external radiotherapy to the recurrent tumor area with dosage of 60 Gy in 2 Gy per fraction. After radiochemotherapy, the obvious reduction of recurrent tumor size was detected. One year later, the patient noticed an augmentation of the mass on her right chest wall. The recurrent tumor was composed with two irregular masses $\left(5.3 \times 5.1 \times 3.0 \mathrm{~cm}^{3}\right.$, and 
$8.0 \times 5.1 \times 4.0 \mathrm{~cm}^{3}$ ) according to the CT scan (see Figure 1). She was referred to our clinic for treatment and requested brachytherapy instead because of the serious skin fibrosis in right chest wall.

Before processing each treatment, informed consent was obtained from the patient. Treatments were performed with standard institutional approval. HDR-interstitial brachytherapy followed by external radiotherapy in total dose of $30 \mathrm{~Gy}$ in 6 fractions during 3 days (5 Gy each fraction, twice a day with 6 hours interval, 3 days) was applied treatment and was accomplished with OncoSmart ProGuide catheters (Nucletron, an Elekta company, Elekta AB, Stockholm, Sweden). Treatment was per-

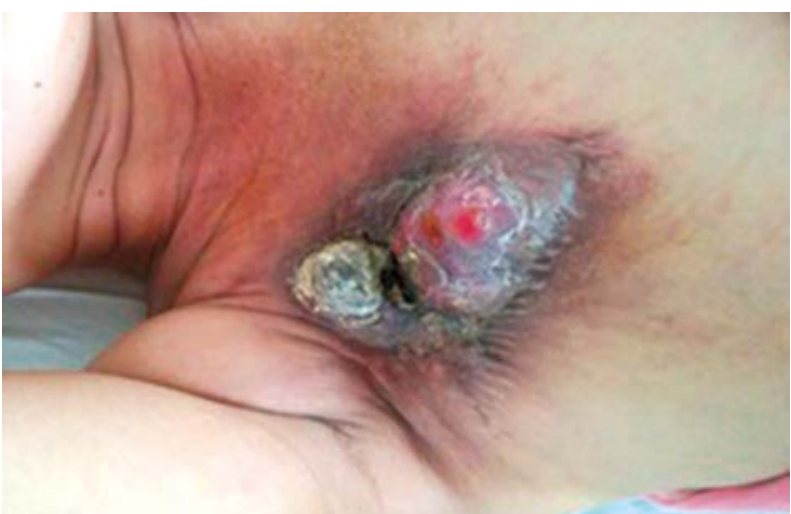

Fig. 1. Recurrent tumor on the skin surface of right chest wall
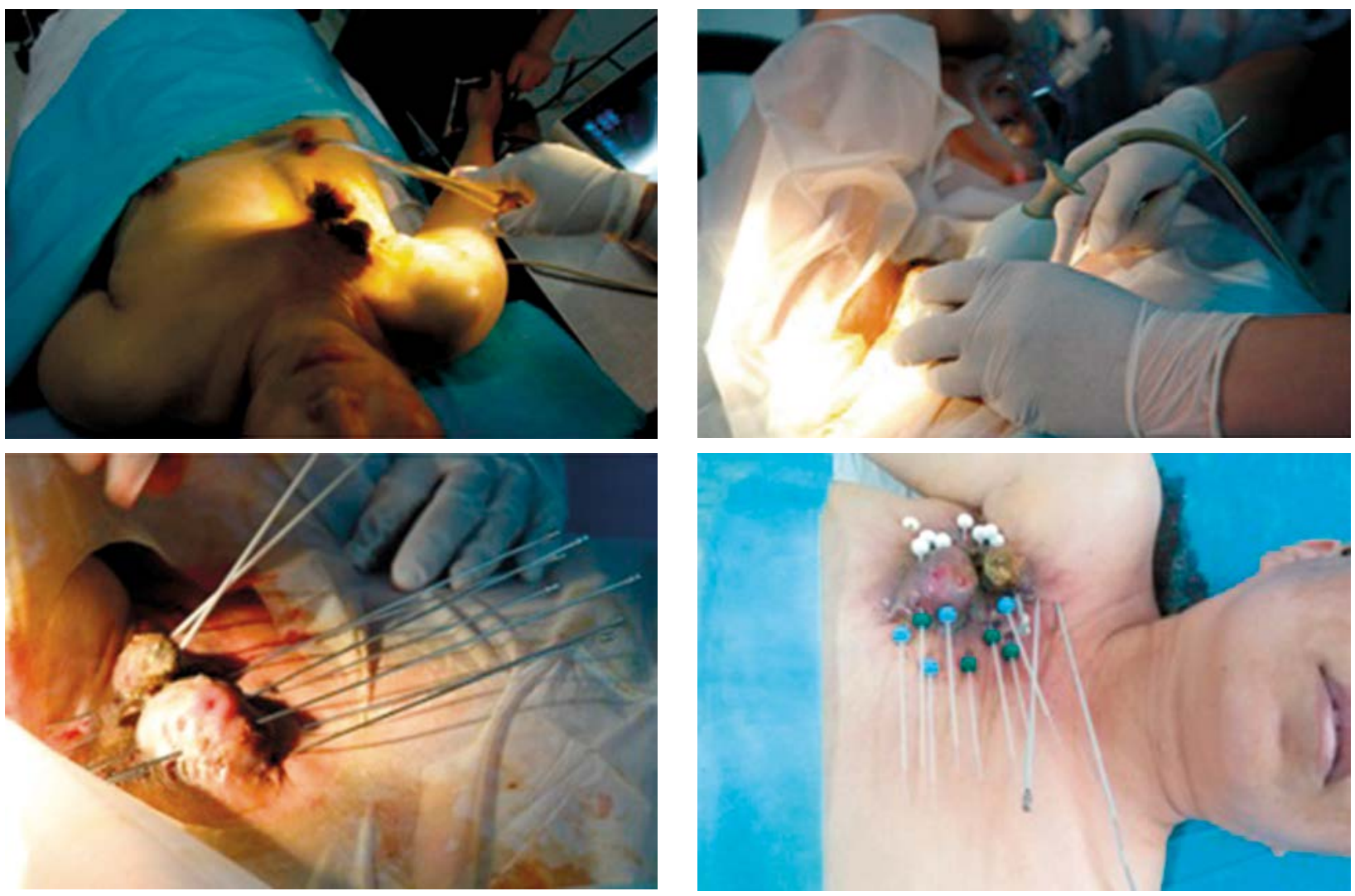

Fig. 2. Eleven applicator needles were inserted to the target under ultrasound guidance 

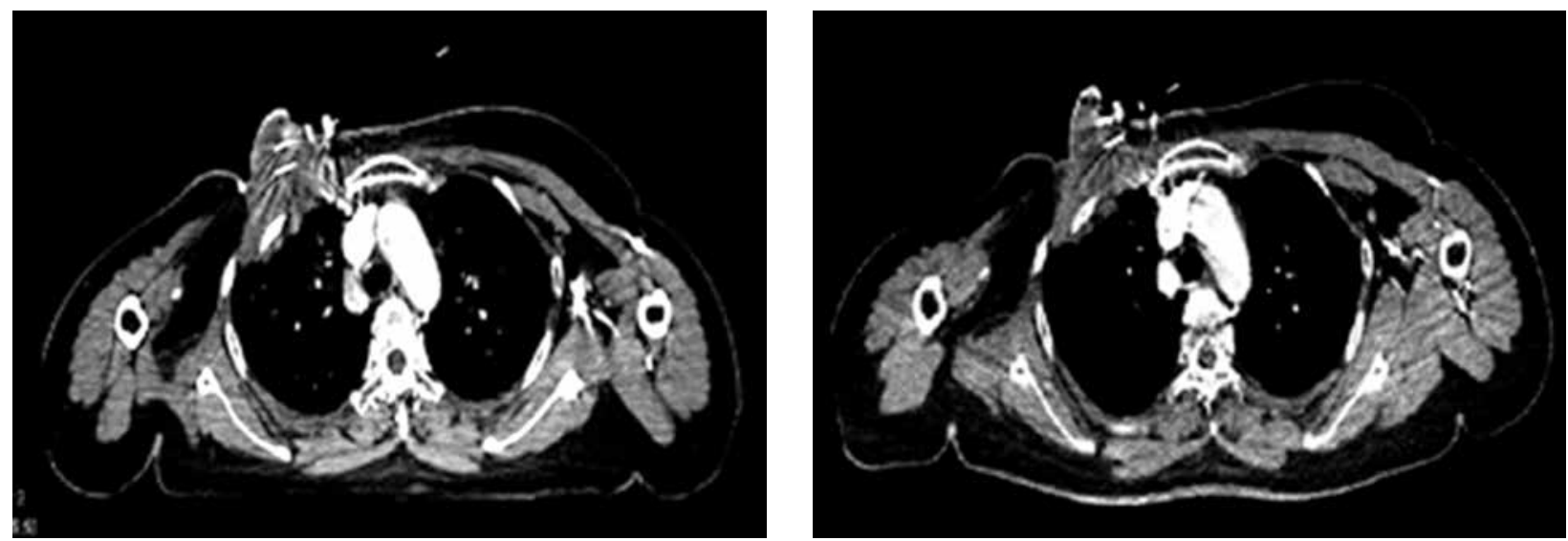

Fig. 3. The position of applicator needles in CT images
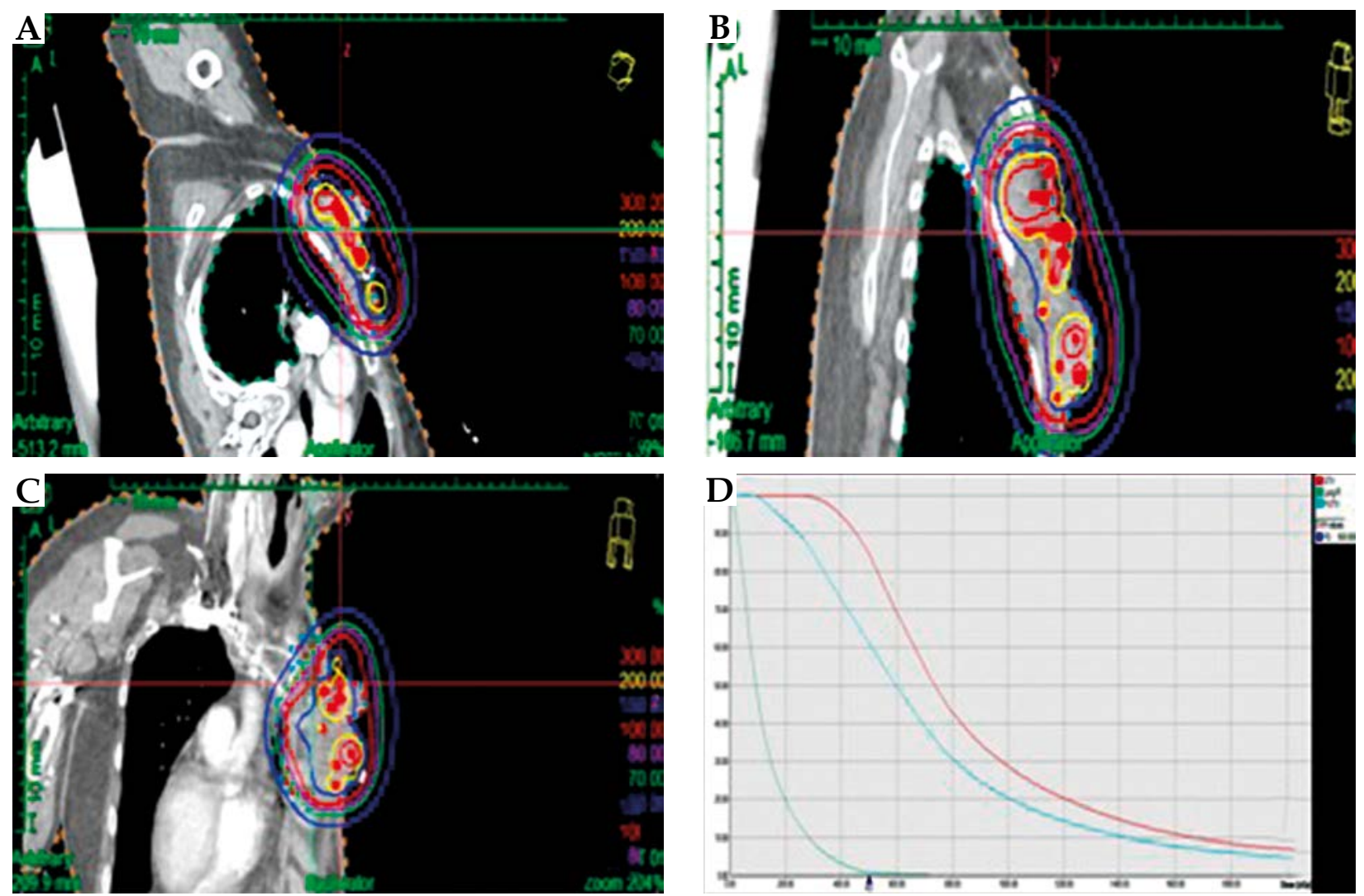

Fig. 4. A-C) The dose distribution of horizontal, saggital and coronal positions. The red line showed the position of $\mathrm{V}_{100}$, covered GTV completely. Just a small part of $\mathrm{V}_{50}$ (showed with the blue line) covered the right lung. D) The dose volume histogram (DVH) parameters. It showed the high dose distribution of GTV and CTV, and low dose distribution of right lung

ume of critical organs (especially right lung). A CT-based treatment plan was created using a graphic optimization tool (Treatment Planning System Oncentra V4.3; Nucletron, Veenendaal, The Netherlands). The normalization and optimization to the target volume was performed.

The dose volume histogram (DVH) parameters are recommended for the evaluation of target volume and organs at risk (OARs). The dose distribution to target volume and OARs are showed in Figure 4. In the brachytherapy plan, $30 \mathrm{~Gy}$ and was prescribed to $100 \%$ of the target volume, and $\mathrm{V}_{20}$ (volume to the most irradiated dose of $20 \mathrm{~Gy}$ ) of the lung was mainly considered. In the external radiotherapy plan, 60 Gy in 2 Gy per fraction was prescribed to the target one year before brachytherapy. The equivalent dose for a 2 Gy fraction schedule was calculated using the EQD2 model, at $\alpha / \beta=3\left(\mathrm{~Gy}_{\mathrm{EQD} 2, \alpha / \beta=3}\right)$ for the lung and $\alpha / \beta=10\left(\mathrm{~Gy}_{\mathrm{EQD} 2, \alpha / \beta=10}\right)$ for the target. Dose volume histogram parameters were analyzed taking into account the volume ratio of the target receiving $90 \%$ or $150 \%$ of the therapeutic dose $\left(\mathrm{V}_{90 \%}\right.$ and $\mathrm{V}_{150 \%}$, 

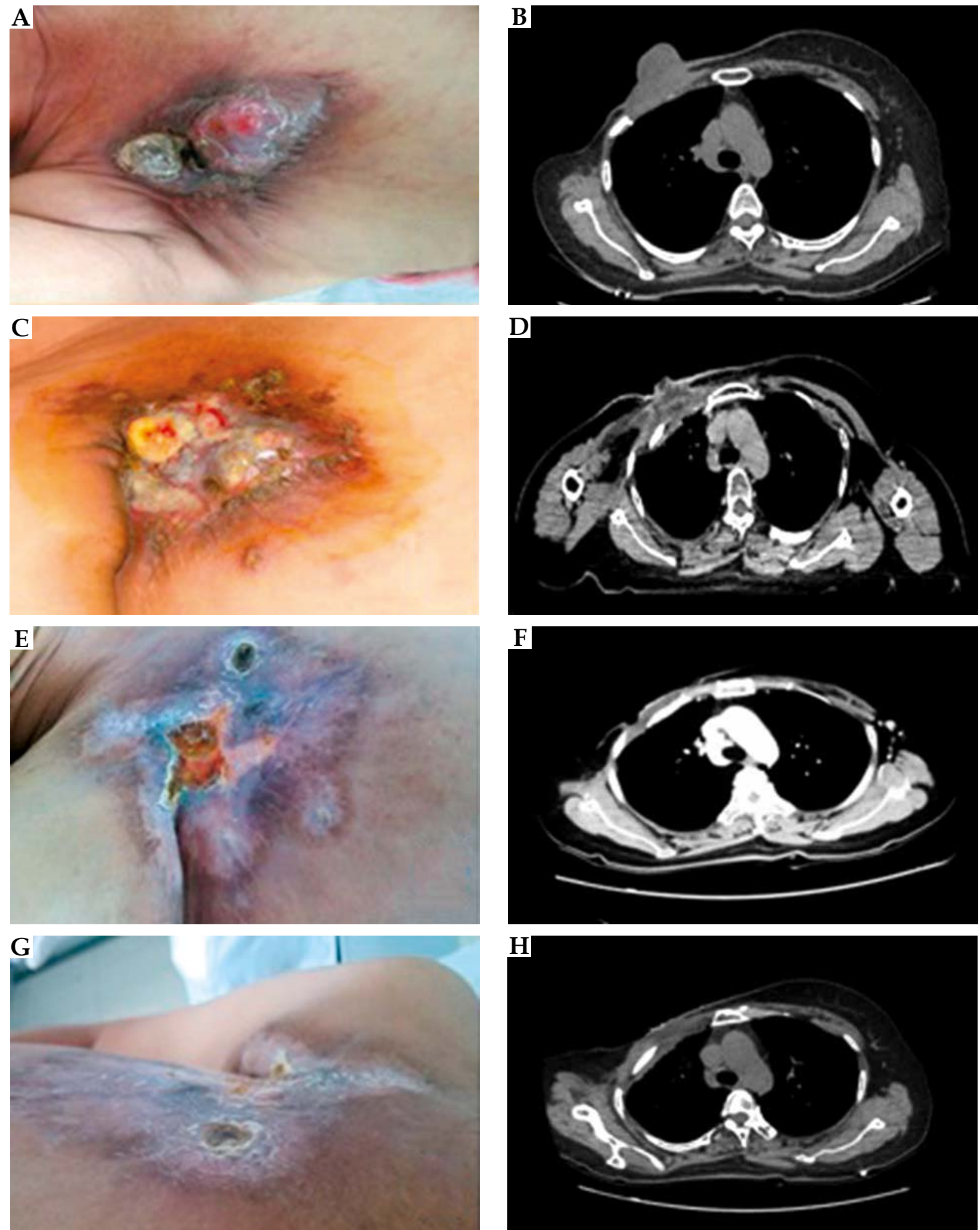

Fig. 5. The right chest wall (A, B) before, (C, D) 2 weeks, (E, F) 3 months and (G, H) 7 months after brachytherapy

respectively), the equivalent dose delivered to $98 \%$ and $50 \%$ of the target volume: $\mathrm{D}_{98 \%}$ and $\mathrm{D}_{50 \%}$, the volume ratio of the lung receiving 5, 10, 20,30, 40, and 50 Gy of the equivalent dose: $\mathrm{V}\left(5 \mathrm{~Gy}_{\mathrm{EQD} 2}\right), \mathrm{V}\left(10 \mathrm{G} \mathrm{y}_{\mathrm{EQD} 2}\right), \mathrm{V}\left(20 \mathrm{~Gy}_{\mathrm{EQD} 2}\right)$,
$\mathrm{V}\left(30 \mathrm{G} \mathrm{y}_{\mathrm{EQD} 2}\right), \mathrm{V}\left(40 \mathrm{G} \mathrm{y}_{\mathrm{EQD} 2}\right)$ and $\mathrm{V}\left(50 \mathrm{G} \mathrm{y}_{\mathrm{EQD} 2}\right)$. The following values were accepted: below $\mathrm{V}_{90 \%}$ was $8.0 \%, \mathrm{~V}_{150 \%}$ was $48.8 \%$; $\mathrm{D}_{98 \%}$ was $29.0 \mathrm{~Gy}, \mathrm{D}_{50 \%}$ was $110.8 \mathrm{~Gy} ; \mathrm{V}\left(5 \mathrm{~Gy} \mathrm{EQD}_{\mathrm{E} 2}\right)$ was $44.9 \%, \mathrm{~V}\left(10 \mathrm{G} \mathrm{y}_{\mathrm{EQD} 2}\right)$ was $27.8 \%, \mathrm{~V}\left(20 \mathrm{~Gy}_{\mathrm{EQD} 2}\right)$ was 
$17.0 \%, \mathrm{~V}\left(30 \mathrm{~Gy}_{\mathrm{EQD} 2}\right)$ was $11.9 \%, \mathrm{~V}\left(40 \mathrm{~Gy}_{\mathrm{EQD} 2}\right)$ was $9.1 \%$, $\mathrm{V}\left(50 \mathrm{~Gy} \mathrm{EQD} 2_{\mathrm{E} 2}\right)$ was $5.6 \%$.

After transporting the planning data to an ${ }^{192} \mathrm{Ir}$ remote afterloader system (Microselectron HDR ${ }^{192} \mathrm{Ir}$; Nucletron, Veenendaal, The Netherlands), irradiation had started. The irradiation took approximately $5 \mathrm{~min}$. The catheters were removed after irradiation was complete, and the patient was discharged after 2 hours under observation. No complications were reported during the treatment and the brachytherapy was well tolerated by the patient. The patient is regularly followed up at our affiliated clinics.

\section{Results}

The CT examination taken 2 weeks after the brachytherapy showed reduction of the tumor size to $4.0 \times$ $1.5 \times 2.7 \mathrm{~cm}^{3}$ and $6.5 \times 1.8 \times 4.5 \mathrm{~cm}^{3}$. During the follow-up examination in the $3^{\text {rd }}$ month after brachytherapy, it was showed that the right chest wall mass had disappeared according to the $\mathrm{CT}$ results. At the present 7 months after reirradiation, treatment-related skin lesions of right chest wall has completely healed (see Figure 5). And there are no signs or symptoms of pectoral complications and no evidence of recurrence at the site of reirradiation.

\section{Discussion}

Although the locoregional recurrence in the breast cancer patients after mastectomy may be a subclinical disseminated disease and may augur poor prognosis, several studies have demonstrated that some of the patients can belong to the favorable subgroup and show long-term survival after salvage treatment [6]. Various treatment strategies including locoregional therapy (wide excision and/or radiotherapy) and/or systemic therapy (hormone therapy and/or chemotherapy) can be considered to treat the breast cancer patients with locoregional recurrence [7]. Hyperthermia is one of the cancer therapies and is considered to be an effective radiation sensitizer [8]. The numerous clinical researches have shown that the addition of hyperthermia to radiation can increase the response rate for breast cancer $[9,10]$. Arunachalam et al. [11] administered combined simultaneous thermobrachytherapy for diffuse chest wall recurrences. However, the efficacy and side effects of combined treatment remain to be observed. The optimal treatment for refractory recurrence of breast cancer is not clearly defined yet. In the present case, the interstitial brachytherapy with good adherence was used for this patient in order to control the recurrent tumor. Eleven applicator catheters were inserted with the guidance of ultrasound. And the DVH showed that GTV was covered with high dose irradiation but the dosage of right lung was relative low. The patient with locoregional recurrence after mastectomy obtained good locoregional control by the interstitial radiotherapy with ultrasound guidance. The entire tumor disappeared 3 months later. And no serious complications were found in follow-up over 7 months after reirradiation. HDR-interstitial brachytherapy provided a superior therapeutic ratio compared with external radiotherapy, and enabled curative dose treatment with prominent therapeutic enhancement.

\section{Conclusions}

Interstitial brachytherapy might be the safe and practicable supplementary method of EBRT. The main benefit of breast brachytherapy compared to EBRT is that a high dose of radiation can be precisely applied to the tumor while sparing radiation to healthy tissues simultaneously. The ultrasound-guided interstitial brachytherapy may be effective for refractory recurrence of breast cancer.

\section{Acknowledgements}

This study was partially supported by a grant from the National Natural Science Foundation of China (81201737) and Bethune Medical Research Program (201310724).

\section{Disclosure}

Authors report no conflict of interest.

\section{References}

1. Saini KS, Taylor C, Ramirez AJ et al. Role of the multidisciplinary team in breast cancer management: results from a large international survey involving 39 countries. Ann Oncol 2012; 23: 853-859.

2. Vaidya JS, Joseph DJ, Tobias JS et al. Targeted intraoperative radiotherapy versus whole breast radiotherapy for breast cancer (TARGIT-A trial): an international, prospective, randomized, non-inferiority phase 3 trial. Lancet 2010; 376: 91-102.

3. Polgár C, Major T. Current status and perspectives of brachytherapy for breast cancer. Int J Clin Oncol 2009; 14: 7-24.

4. Guinot JL, Baixauli-Perez C, Soler P et al. High-dose-rate brachytherapy boost effect on local tumor control in young women with breast cancer. Int J Radiat Oncol Biol Phys 2015; 91: 165-171.

5. Kuske R. Breast conservation therapy without capsular contracture in young augmented women using interstitial brachytherapy. J Contemp Brachytherapy 2014; 6: 231-235.

6. Kuo SH, Huang CS, Kuo WH et al. Comprehensive locoregional treatment and systemic therapy for postmastectomy isolated locoregional recurrence. Int J Radiat Oncol Biol Phys 2008; 72: 1456-1464.

7. Jeong Y, Kim SS, Gong G et al. Treatment results of breast cancer patients with locoregional recurrence after mastectomy. Radiat Oncol J 2013; 31: 138-146.

8. Chicheł A, Skowronek J, Kanikowski M. Thermal boost combined with interstitial brachytherapy in breast conserving therapy - assessment of early toxicity. Rep Pract Oncol Radiother 2011; 16: 87-94.

9. Stauffer PR, Maccarini P, Arunachalam K et al. Conformal microwave array (CMA) applicators for hyperthermia of diffuse chest wall recurrence. Int J Hyperthermia 2010; 26: 686-698.

10. Jones EL, Marks LB, Prosnitz LR. Point: Hyperthermia with radiation for chest wall recurrences. J Natl Compr Canc Netw 2007; 5: 339-344.

11. Arunachalam K, Maccarini PF, Craciunescu OI et al. Thermal characteristics of thermobrachytherapy surface applicators for treating chest wall recurrence. Phys Med Biol 2010; 55: 1949-1969. 